\title{
Uplink Power Efficiency of Multiuser MIMO with Very Large Antenna Arrays
}

Quoc Hien Ngo, Erik G. Larsson and Thomas L. Marzetta

\section{Linköping University Post Print}

N.B.: When citing this work, cite the original article.

(C2011 IEEE. Personal use of this material is permitted. However, permission to reprint/republish this material for advertising or promotional purposes or for creating new collective works for resale or redistribution to servers or lists, or to reuse any copyrighted component of this work in other works must be obtained from the IEEE.

Quoc Hien Ngo, Erik G. Larsson and Thomas L. Marzetta, Uplink Power Efficiency of Multiuser MIMO with Very Large Antenna Arrays, 2011, Proceedings of Allerton Conference on Communication, Control, and Computing. 


\title{
Uplink Power Efficiency of Multiuser MIMO with Very Large Antenna Arrays
}

\author{
Hien Quoc Ngo*, Erik G. Larsson* and Thomas L. Marzetta ${ }^{\dagger}$ \\ *Department of Electrical Engineering (ISY) \\ Linköping University, 58183 Linköping, Sweden \\ ${ }^{\dagger}$ Bell Laboratories, Alcatel-Lucent \\ Murray Hill, NJ 07974, USA
}

\begin{abstract}
A multiplicity of autonomous terminals simultaneously transmits data streams to a compact array of antennas. The array uses imperfect channel-state information derived from transmitted pilots to extract the individual data streams. The power radiated by the terminals can be made inversely proportional to the square-root of the number of base station antennas with no reduction in performance. In contrast if perfect channel-state information were available the power could be made inversely proportional to the number of antennas. A maximum-ratio combining receiver normally performs worse than a zero-forcing receiver. However as power levels are reduced, the cross-talk introduced by the inferior maximum-ratio receiver eventually falls below the noise level and this simple receiver becomes a viable option.
\end{abstract}

\section{INTRODUCTION}

In multiuser multiple-input multiple-output (MU-MIMO) systems, a base station equipped with multiple antennas serves a number of users. Each user may be equipped with a single or multiple antennas. Such systems have attracted a lot of attention for a number of years now [1]. Conventionally, the communication between the base station and the users is performed by orthogonalizing the channel so that the base station communicates with each user in separate timefrequency resources. This is not optimal from an informationtheoretic point of view, and it is better if the base station communicates with several users in the same time-frequency resource [2], [3]. However, complex techniques to mitigate or reduce the interference from other users must then be used, such as maximum-likelihood multiuser detection on the uplink [4], or "dirty-paper coding" on the downlink [5], [6].

Recently, there has been a great deal of interest in MUMIMO with very large antenna arrays at the base station. Very large arrays can substantially reduce intracell interference with simple signal processing [7]. We refer to such systems as "very large MU-MIMO systems" here, and with very large arrays we mean arrays comprising say a hundred, or a few hundreds, of antennas. The design and analysis of very large MU-MIMO systems is a fairly new subject that is attracting substantial interest [7]-[9]. The vision is that each individual antenna in such a system would be an active antenna which has a small physical size, and can be built from inexpensive hardware. With a very large antenna array, things that were random before start to look deterministic. As a consequence, the effect of small-scale fading can be averaged out. Furthermore, from the law of large numbers, when the number of base station antennas grows large, the random channel vectors between the users and the base station become pairwisely orthogonal. Therefore, the interference from other users can be canceled without using more time-frequency resources. In [7], a very large multicell MU-MIMO system was studied. The author showed that, with an unlimited number of base station antennas, with simple maximum-ratio combining (MRC) at the receiver and maximum-ratio transmission (MRT) at the transmitter, the effects of fast fading, uncorrelated noise, and intracell interference disappear. Another important advantage of large MIMO systems is that they enable us to reduce the transmitted power. On the uplink, reducing the terminals' transmit power will drain their batteries slower. On the downlink, much of the electrical power consumed by a base station is spent by power amplifiers and associated circuits and cooling systems. Hence reducing the emitted RF power would help in cutting the electricity consumption of the base station.

This paper analyzes the power efficiency and the potential for power savings of very large MU-MIMO systems. While it is well known that MIMO technology can offer improved power efficiency, owing to both array gains and diversity effects [10], [11], there is very little, if any, work available that analyzes power efficiency of MU-MIMO systems. We consider the power efficiency of a single cell MU-MIMO system, and we focus on the case where the users have a single antenna each. We present both results for finite-sized antenna arrays and asymptotic results assuming that the number of elements in array is very large. The focus here is on the uplink, leaving a study of the downlink for future work. The basic question that we address is how much the transmitted power of each user can be reduced while ensuring a fixed quality-of-service. The paper makes the following specific contributions:

- We show that, when the number of base station antennas $M$ grows without bound, we can reduce the transmitted power of each user proportionally to $1 / M$ if the base station has perfect channel state information (CSI), and proportionally to $1 / \sqrt{M}$ for the case of imperfect CSI obtained from uplink pilot measurements. This can be accomplished by using simple, linear receivers. See Section III. 
- We derive closed-form expressions of lower bounds on the uplink achievable rates for finite but large $M$, for the cases of perfect and imperfect CSI, assuming MRC and zero-forcing (ZF) receivers, respectively. See Section IV.

Notation: We use upper (lower) bold letters to denote matrices (vectors). The superscripts $T$, *, and $H$ stand for the transpose, conjugate, and conjugate-transpose, respectively. $[\boldsymbol{A}]_{i j}$ or $\boldsymbol{A}_{i j}$ denotes the $(i, j)$ th entry of a matrix $\boldsymbol{A}$, and $\boldsymbol{I}_{n}$ is the $n \times n$ identity matrix. The expectation operation and the Euclidean norm are denoted by $\mathbb{E}\{\cdot\}$ and $\|\cdot\|$, respectively. Finally, we use $z \sim \mathcal{C N}(0, \Sigma)$ to denote a circularly symmetric complex Gaussian vector $z$ with covariance matrix $\Sigma$ and zero mean.

\section{System Model AND PRELIMinaries}

\section{A. MU-MIMO System Model}

We consider a MU-MIMO system which includes one base station equipped with $M$ antennas serving $K$ single-antenna users. We consider uplink transmission. The communication between the base station and the users takes place in the same time-frequency resource. The system that we consider is single-cell, or equivalently, it is a multicell system operating in the noise-limited regime.

The $M \times 1$ received vector at the base station is given by

$$
\boldsymbol{y}=\sqrt{p_{\mathrm{u}}} \boldsymbol{G} \boldsymbol{x}+\boldsymbol{n}
$$

where $G$ represents the $M \times K$ channel matrix between the base station and the $K$ users, i.e., $g_{m k} \triangleq[G]_{m k}$ is the channel coefficient between the $m$ th antenna of the base station and the $k$ th user; $\sqrt{p_{\mathrm{u}}} x$ is the $K \times 1$ transmitted vector of $K$ users (the average transmitted power of each user is $p_{\mathrm{u}}$ ); and $n$ is an $M \times 1$ additive white Gaussian noise (AWGN) vector whose elements are independent with zero mean and variance $\sigma^{2}$.

The channel matrix $\boldsymbol{G}$ models independent fast fading, geometric attenuation, and log-normal shadow fading. Then, $g_{m k}$ can be represented as

$$
g_{m k}=h_{m k} \sqrt{\beta_{k}}, \quad m=1,2, \ldots, M
$$

where $h_{m k}$ is the fast fading coefficient from the $k$ th user to the $m$ th antenna of the base station. We assume that the coefficient $h_{m k}$ has zero mean and unit variance. Furthermore, $\sqrt{\beta_{k}}$ models the geometric attenuation and shadow fading which are assumed to be independent over $m$ and to be constant over many coherence time intervals and known a priori. This assumption is reasonable since the distance between the user and the base station is much larger than the distance between the antennas, and the value of $\beta_{k}$ changes very slowly with time. Then, we have

$$
G=H D^{1 / 2}
$$

where $H$ is the $M \times K$ matrix of fast fading coefficients between the $K$ users and the base station, i.e., $[\boldsymbol{H}]_{m k}=h_{m k}$, and $\boldsymbol{D}$ is a $K \times K$ diagonal matrix whose diagonal elements are given by $[\boldsymbol{D}]_{k k}=\beta_{k}$. Therefore, (1) can be written as

$$
\boldsymbol{y}=\sqrt{p_{\mathrm{u}}} \boldsymbol{H} \boldsymbol{D}^{1 / 2} \boldsymbol{x}+\boldsymbol{n} .
$$

\section{B. Review of Some Results on Very Long Random Vectors}

Before we proceed to analyzing the MU-MIMO model defined in Section II-A, we review some useful limit results about very long random vectors [12] .

- Let $\boldsymbol{p} \triangleq\left[\begin{array}{lll}p_{1} & \ldots & p_{n}\end{array}\right]^{T}$ and $\boldsymbol{q} \triangleq\left[\begin{array}{lll}q_{1} & \ldots & q_{n}\end{array}\right]^{T}$ be $n \times 1$ vectors whose elements are independent identically distributed (i.i.d.) random variables (RVs) with $\mathbb{E}\left\{p_{i}\right\}=\mathbb{E}\left\{q_{i}\right\}=0$, $\mathbb{E}\left\{\left|p_{i}\right|^{2}\right\}=\sigma_{p}^{2}$, and $\mathbb{E}\left\{\left|q_{i}\right|^{2}\right\}=\sigma_{q}^{2}, i=1,2, \ldots, n$. Assume that $\boldsymbol{p}$ and $\boldsymbol{q}$ are independent.

Applying the law of large numbers, we obtain

$$
\begin{aligned}
& \frac{1}{n} \boldsymbol{p}^{H} \boldsymbol{p} \stackrel{\text { a.s. }}{\rightarrow} \sigma_{p}^{2}, \text { as } n \rightarrow \infty \\
& \frac{1}{n} \boldsymbol{p}^{H} \boldsymbol{q} \stackrel{\text { a.s. }}{\rightarrow} 0, \text { as } n \rightarrow \infty .
\end{aligned}
$$

where $\stackrel{\text { a.s. }}{\rightarrow}$ denotes the almost sure convergence.

Applying Lindeberg-Lévy central limit theorem, we obtain

$$
\frac{1}{\sqrt{n}} \boldsymbol{p}^{H} \boldsymbol{q} \stackrel{d}{\rightarrow} \mathcal{C N}\left(0, \sigma_{p}^{2} \sigma_{q}^{2}\right), \text { as } n \rightarrow \infty
$$

where $\stackrel{d}{\rightarrow}$ denotes convergence in distribution.

- Let $X_{1}, X_{2}, \ldots$ be a sequence of independent RVs, such that $X_{i}$ has zero mean and variance $\sigma_{i}^{2}$. Further assume that the following conditions are satisfied: 1) $s_{n}^{2}=\sum_{i=1}^{n} \sigma_{i}^{2} \rightarrow \infty$, as $n \rightarrow \infty$; and 2) $\sigma_{i} / s_{n} \rightarrow 0$, as $n \rightarrow \infty$. Then by applying the Cramér's central limit theorem [12], we have

$$
\frac{\sum_{i=1}^{n} X_{i}}{s_{n}} \rightarrow \mathcal{C N}(0,1), \text { as } n \rightarrow \infty .
$$

These results will be used for the analysis of the power efficiency in the next section.

\section{Asymptotic $(M \rightarrow \infty)$ POWER EFFiCIENCY}

The potential for improved power efficiency is one of the most important advantages of very large MIMO technology. By using a large antenna array, we can reduce the transmitted power of the users with a factor of $M^{\alpha}$ while maintaining a given, desired quality-of-service as $M$ grows large. In this section, we will compute the largest possible $\alpha$ under the assumptions of perfect and imperfect transmitter CSI, respectively. The goal of this analysis is to provide basic insights into the scaling laws that govern power-efficiency of MU-MIMO in the very-large- $M$ regime. More precise results for finite $M$, which can be used for accurate evaluation of power savings but which have a less intuitive form, will be derived in Section IV.

\section{A. Perfect Channel State Information}

We first consider the case when the base station has perfect CSI, i.e. it perfectly knows $G$. Theoretically, the base station can use the maximum-likelihood detector to obtain optimal performance. However, the complexity of this detector grows exponentially with the number of users. The interesting operating regime is when both $M$ and $K$ are large, but $M$ is still 
(much) larger than $K$, i.e., $1 \ll K \ll M$. It is known that in this case, linear detectors perform fairly well [7] and therefore we will restrict consideration to such detectors in this paper. Specifically, we consider the ZF receiver. By using ZF, the received signal is separated into streams, which are decoded independently. The separation into streams is accomplished by multiplying with the pseudo inverse of $\boldsymbol{G}$ as follows

$$
\begin{aligned}
\boldsymbol{r}_{\mathrm{ZF}} & =\boldsymbol{G}^{\dagger} \boldsymbol{y} \\
& =\boldsymbol{G}^{\dagger}\left(\sqrt{p_{\mathrm{u}}} \boldsymbol{G} \boldsymbol{x}+\boldsymbol{n}\right)
\end{aligned}
$$

where $G^{\dagger} \triangleq\left(G^{H} G\right)^{-1} G^{H}$. Notice that, from (3), (5) and (6), when $M$ grows large, $\frac{1}{M} G^{H} \boldsymbol{G}$ tends to $\boldsymbol{D}$ and hence, $G^{\dagger}$ tends to $\frac{1}{M} \boldsymbol{D}^{-1} \boldsymbol{G}^{H}$ which corresponds to the MRC. ${ }^{1}$ For this reason, and since we are considering very large antenna arrays, in this section, we will consider the MRC receiver instead of the ZF receiver. (In Section IV we consider the case of finite $M$, and there we distinguish between the MRC and ZF receivers.) The received signal after using MRC is given by

$$
\boldsymbol{r}_{\mathrm{MRC}}=\boldsymbol{G}^{H} \boldsymbol{y} .
$$

If $K=1$, then it is clear that we can scale down the transmit power by a factor $1 / M$ without compromising the qualityof-service. This factor is simply the array gain of the MRC receiver. The following proposition shows that as $M \rightarrow \infty$, this scaling law is valid also in a multiuser system, i.e., for $K$ fixed, and $K>1$.

Proposition 1: Assume that the base station has perfect CSI and that the transmit power of each user is

$$
p_{\mathrm{u}}=\frac{E_{\mathrm{u}}}{M}
$$

where $E_{\mathrm{u}}$ is fixed. Further, assume that the base station uses MRC. (This is equivalent to $\mathrm{ZF}$ in the limit of $M \rightarrow \infty$, as discussed above.) Let $\operatorname{SINR}_{k}^{\mathrm{P}}$ be the signal-to-interferenceplus-noise ratio (SINR) of the uplink transmission from the $k$ th user. Then,

$$
\operatorname{SINR}_{k}^{\mathrm{P}} \rightarrow \frac{\beta_{k} E_{\mathrm{u}}}{\sigma^{2}}, M \rightarrow \infty .
$$

In the limit, there is no interference and hence the SINR will be equal to the SNR.

Proof: With $p_{\mathrm{u}}=\frac{E_{\mathrm{u}}}{M}$, by using MRC, the processed received signal becomes

$$
r_{\mathrm{MRC}}=G^{H}\left(\sqrt{\frac{E_{\mathrm{u}}}{M}} G \boldsymbol{x}+\boldsymbol{n}\right) .
$$

We have

$$
\frac{1}{\sqrt{M}} \boldsymbol{r}_{\mathrm{MRC}}=\sqrt{E_{\mathrm{u}}} \frac{\boldsymbol{G}^{H} \boldsymbol{G}}{M} x+\frac{1}{\sqrt{M}} \boldsymbol{G}^{H} \boldsymbol{n}
$$

\footnotetext{
${ }^{1}$ The fact that the propagation vectors for different users become asymptotically orthogonal when $M$ grows large holds under many propagation conditions [7].
}

From (5)-(7), when $M$ grows large, we obtain

$$
\frac{1}{\sqrt{M}} \boldsymbol{r}_{\mathrm{MRC}} \rightarrow \sqrt{E_{\mathrm{u}}} \boldsymbol{D} \boldsymbol{x}+\boldsymbol{D}^{1 / 2} \tilde{\boldsymbol{n}}
$$

where $\tilde{\boldsymbol{n}} \sim \mathcal{C N}\left(0, \sigma^{2} \boldsymbol{I}\right)$. It follows that the SINR of the uplink transmission from the $k$ th user is given by (11).

Proposition 1 shows that with perfect CSI at the base station and a large $M$, the performance of a MU-MIMO system with $M$ antennas at the base station and a transmit power per user equal to $E_{\mathrm{u}} / M$ is equal to the performance of a SISO system with transmit power $E_{\mathrm{u}}$, without any intra-cell interference and without any fast fading. In other words, by using a large number of base station antennas, we can scale down the transmit power by a factor of $1 / M$, as well increase the spectral efficiency $K$ times (since we do not need to assign different frequencies or time-slots to different users).

\section{B. Imperfect Channel State Information}

In practice, the channels have to be estimated at the base station. The standard way of doing this is to use uplink pilots. A part of the coherence interval of the channel is then used for the uplink training. Let $\tau$ be the number of symbols used entirely for pilots. All users simultaneously transmit pilot sequences of length $\tau$ symbols. The pilot sequences of users are pairwise orthogonal. Therefore, the pilot sequences used by the $K$ users can be represented by a $\tau \times K$ matrix $\sqrt{p_{\mathrm{p}}} \boldsymbol{\Phi}$ $(\tau \geq K)$, which satisfies $\boldsymbol{\Phi}^{H} \boldsymbol{\Phi}=\boldsymbol{I}_{K}$, where $p_{\mathrm{p}}=\tau p_{\mathrm{u}}$. Then, the $M \times \tau$ received pilot matrix at the base station is given by

$$
\boldsymbol{Y}_{\mathrm{p}}=\sqrt{p_{\mathrm{p}}} \boldsymbol{G} \boldsymbol{\Phi}^{T}+\boldsymbol{N}
$$

where $N$ is the $M \times \tau$ complex AWGN matrix whose elements are independent with zero mean and variance $\sigma^{2}$. Then, the minimum-mean-square-error (MMSE) estimate of $\boldsymbol{G}$ is given by

$$
\hat{\boldsymbol{G}}=\frac{1}{\sqrt{p_{\mathrm{p}}}} \boldsymbol{Y}_{\mathrm{p}} \boldsymbol{\Phi}^{*} \tilde{D}=\left(\boldsymbol{G}+\frac{1}{\sqrt{p_{\mathrm{p}}}} \boldsymbol{W}\right) \tilde{\boldsymbol{D}}
$$

where $\boldsymbol{W} \triangleq N \Phi^{*}$, and $\tilde{\boldsymbol{D}} \triangleq\left(\frac{\sigma^{2}}{p_{\mathrm{p}}} \boldsymbol{D}^{-1}+\boldsymbol{I}_{K}\right)^{-1}$. Since $\boldsymbol{\Phi}^{H} \boldsymbol{\Phi}=\boldsymbol{I}_{K}, \boldsymbol{W}$ is an $M \times K$ random matrix whose elements are i.i.d. zero-mean complex Gaussian with variance $\sigma^{2}$.

If we cut the transmitted power of each user to $\frac{E_{u}}{M}$ as in the perfect-CSI case, the processed signal vector at the base station after using MRC (with $G$ replaced by $\hat{G}$ ) is given by

$$
\begin{aligned}
\hat{\boldsymbol{r}}_{\mathrm{MRC}} & =\hat{\boldsymbol{G}}^{H} \boldsymbol{y} \\
& =\tilde{\boldsymbol{D}}\left(\boldsymbol{G}^{H}+\frac{\sqrt{M}}{\sqrt{\tau E_{\mathrm{u}}}} W^{H}\right)\left(\sqrt{\frac{E_{\mathrm{u}}}{M}} G \boldsymbol{x}+\boldsymbol{n}\right) .
\end{aligned}
$$

Therefore,

$$
\begin{aligned}
\frac{1}{\sqrt{M}} \tilde{\boldsymbol{D}}^{-1} \hat{\boldsymbol{r}}_{\mathrm{MRC}}= & \sqrt{E_{\mathrm{u}}} \frac{\boldsymbol{G}^{H} \boldsymbol{G}}{M} \boldsymbol{x}+\frac{1}{\sqrt{M}} \boldsymbol{G}^{H} \boldsymbol{n} \\
& +\frac{1}{\sqrt{\tau M}} \boldsymbol{W}^{H} \boldsymbol{G} \boldsymbol{x}+\frac{1}{\sqrt{\tau E_{\mathrm{u}}}} \boldsymbol{W}^{H} \boldsymbol{n} .
\end{aligned}
$$


From (5)-(7), as $M$ grows large, we obtain

$$
\begin{aligned}
\frac{1}{\sqrt{M}} \tilde{\boldsymbol{D}}^{-1} \hat{\boldsymbol{r}}_{\mathrm{MRC}} \rightarrow & \sqrt{E_{\mathrm{u}}} \boldsymbol{D} \boldsymbol{x}+\boldsymbol{D}^{1 / 2} \tilde{\boldsymbol{n}} \\
& +\frac{1}{\sqrt{\tau}} \tilde{\boldsymbol{W}} \boldsymbol{D}^{1 / 2} \boldsymbol{x}+\frac{\sqrt{M}}{\sqrt{\tau E_{\mathrm{u}}}} \tilde{\boldsymbol{w}}
\end{aligned}
$$

where $\tilde{\boldsymbol{w}} \sim \mathcal{C N}\left(0, \sigma^{4} \boldsymbol{I}\right)$. The elements of the $M \times K$ matrix $\tilde{W}$ are independent complex Gaussian RVs with zero means and variances $\sigma^{2}$. We can see that the last term is a noise component which goes to infinity as $M \rightarrow \infty$ and hence the SINR goes to zero. This means that we cannot cut the transmitted power at each user proportionally to $1 / M$, as in the case of perfect CSI.

Intuitively, if we cut the transmitted power of the each user, both the data signal and the pilot signal suffer from the reduction in power. Hence there is a "squaring effect". As a consequence, we can only cut the transmitted power by a smaller factor. The following proposition shows that it is possible to reduce the power proportionally to $1 / \sqrt{M}$.

Proposition 2: Assume that the base station has imperfect CSI, obtained by MMSE estimation from uplink pilots. Further assume that the transmit power of each user is

$$
p_{\mathrm{u}}=\frac{E_{\mathrm{u}}}{\sqrt{M}}
$$

and that the base station uses MRC. (We can show that this is equivalent to $\mathrm{ZF}$ in the limit of $M \rightarrow \infty$ by using a similar argument as in discussion in the case of perfect CSI.) Then the SINR of the uplink transmission for the $k$ th user, $\operatorname{SINR}_{k}^{\mathrm{IP}}$, behaves as

$$
\operatorname{SINR}_{k}^{\mathrm{IP}} \rightarrow \frac{\tau \beta_{k}^{2} E_{\mathrm{u}}^{2}}{\sigma^{4}}, \text { as } M \rightarrow \infty .
$$

Proof: With $p_{\mathrm{u}}=\sqrt{\frac{E_{\mathrm{u}}}{M}}$, we have

$$
\hat{\boldsymbol{r}}_{\mathrm{MRC}}=\tilde{\boldsymbol{D}}\left(\boldsymbol{G}^{H}+\frac{\sqrt[4]{M}}{\sqrt{\tau E_{\mathrm{u}}}} \boldsymbol{W}^{H}\right)\left(\frac{\sqrt{E_{\mathrm{u}}}}{\sqrt[4]{M}} G \boldsymbol{x}+\boldsymbol{n}\right) .
$$

Therefore,

$$
\begin{aligned}
\frac{1}{\sqrt[4]{M^{3}}} \tilde{\boldsymbol{D}}^{-1} \hat{\boldsymbol{r}}_{\mathrm{MRC}}= & \sqrt{E_{\mathrm{u}}} \frac{\boldsymbol{G}^{H} \boldsymbol{G}}{M} \boldsymbol{x}+\frac{1}{\sqrt[4]{M^{3}}} \boldsymbol{G}^{H} \boldsymbol{n} \\
& +\frac{1}{\sqrt{\tau}} \frac{\boldsymbol{W}^{H} \boldsymbol{G}}{\sqrt[4]{M^{3}}} x+\frac{1}{\sqrt{\tau E_{\mathrm{u}}}} \frac{\boldsymbol{W}^{H} \boldsymbol{n}}{\sqrt{M}} .
\end{aligned}
$$

From (5)-(7), as $M$ grows large, we obtain

$$
\frac{1}{\sqrt[4]{M^{3}}} \tilde{\boldsymbol{D}}^{-1} \hat{\boldsymbol{r}}_{\mathrm{MRC}} \rightarrow \sqrt{E_{\mathrm{u}}} \boldsymbol{D} \boldsymbol{x}+\frac{1}{\sqrt{\tau E_{\mathrm{u}}}} \tilde{\boldsymbol{w}}
$$

Therefore, as $M \rightarrow \infty$, the SINR of the uplink transmission from the $k$ th user is given by (20).

Proposition 2 implies that with imperfect CSI at the base station and a large $M$, the performance of a MU-MIMO system with an $M$-antenna base station and with the transmit power per user set to $E_{\mathrm{u}} / \sqrt{M}$ is equal to the performance of an interference-free SISO link with transmit power $\tau \beta_{k} E_{\mathrm{u}}^{2} / \sigma^{2}$, without fast fading.
Remark 1: From the proof of Theorem 2, we can show that if the we cut the transmit power proportionally to $1 / M^{\alpha}$, where $\alpha>1 / 2$, then the SINR of the uplink transmission from the $k$ th user will go to zero when $M \rightarrow \infty$. This means that $\alpha=1 / 2$ is the largest order by which we can cut the transmit power of each user and still guarantee a given qualityof-service.

Remark 2: In general, each user can use different transmit powers which depend on the geometric attenuation and the shadow fading. This can be done by assuming that the $k$ th user knows $\beta_{k}$ and performs power control. Following a similar reasoning as that leading to the result in Proposition 2, we can see that, to achieve the same quality-of-service as a SISO system with the transmit power $E_{\mathrm{u}}$, the transmitted power of the $k$ th user can be chosen as $\sqrt{\frac{\sigma^{2} E_{u}}{M \tau \beta_{k}}}$.

\section{Finite- $M$ AnALYis}

In this section, we consider the case of a finite $M$ (we require $M \geq K$ ) and derive achievable rates of the uplink transmission. This analysis leads to lower bounds on the achievable rate and can be used to draw more precise quantitative conclusions on power efficiency. We treat the cases of perfect and imperfect CSI at the base station, and the cases MRC and ZF receivers. Here, with finite $M, \mathrm{MRC}$ and ZF are not equivalent, unlike in Section III. The lower bounds that we compute in what follows are obtained by assuming that the interference plus noise has a Gaussian distribution [13]. Furthermore, we assume that the fast fading is Rayleigh fading, i.e., the elements of $\boldsymbol{H}$ are i.i.d. Gaussian RVs with zero means and unit variances.

\section{A. Perfect Channel State Information}

1) Maximum-Ratio Combining: For the perfect CSI, after applying MRC, the received signal transmitted from the $k$ th user is

$$
r_{\mathrm{MRC}, k}=\sqrt{p_{\mathrm{u}}}\left\|\boldsymbol{g}_{k}\right\|^{2} x_{k}+\sqrt{p_{\mathrm{u}}} \sum_{i \neq k}^{K} \boldsymbol{g}_{k}^{H} \boldsymbol{g}_{i} x_{i}+\boldsymbol{g}_{k}^{H} \boldsymbol{n}
$$

where $g_{i}$ is the $i$ th column of $G$, and $x_{i}$ is the transmitted signal from the $i$ th user. By dividing the left and right hand sides of (24) by $\left\|\boldsymbol{g}_{k}\right\|$, we obtain

$$
\begin{aligned}
\frac{r_{\mathrm{MRC}, k}}{\left\|\boldsymbol{g}_{k}\right\|} & =\sqrt{p_{\mathrm{u}}}\left\|\boldsymbol{g}_{k}\right\| x_{k}+\sqrt{p_{\mathrm{u}}} \sum_{i \neq k}^{K} \frac{\boldsymbol{g}_{k}^{H} \boldsymbol{g}_{i}}{\left\|\boldsymbol{g}_{k}\right\|} x_{i}+\frac{\boldsymbol{g}_{k}^{H}}{\left\|\boldsymbol{g}_{k}\right\|} \boldsymbol{n} \\
& =\sqrt{p_{\mathrm{u}}}\left\|\boldsymbol{g}_{k}\right\| x_{k}+\sqrt{p_{\mathrm{u}}} \sum_{i \neq k}^{K} \tilde{g}_{i} x_{i}+\tilde{n}_{k}
\end{aligned}
$$

where $\tilde{g}_{i} \triangleq \frac{\boldsymbol{g}_{k}^{H} \boldsymbol{g}_{i}}{\left\|\boldsymbol{g}_{k}\right\|}$, and $\tilde{n}_{k} \triangleq \frac{\boldsymbol{g}_{k}^{H} \boldsymbol{n}}{\left\|\boldsymbol{g}_{k}\right\|}$. Conditioned on $\boldsymbol{g}_{k}, \tilde{g}_{i}$ is a Gaussian $\mathrm{RV}$ with zero mean and variance $\beta_{i}$ which does not depend on $\boldsymbol{g}_{k}$. Therefore, $\tilde{g}_{i}$ is Gaussian distributed and independent of $\boldsymbol{g}_{k}, \tilde{g}_{i} \sim \mathcal{C N}\left(0, \beta_{i}\right)$. Similarly, we have $\tilde{n}_{k} \sim$ $\mathcal{C N}\left(0, \sigma^{2}\right)$ which is independent of $\boldsymbol{g}_{k}$.

Assume that the base station has only knowledge of $\boldsymbol{g}_{k}$, and that the term which includes the interference and noise is 


$$
\mathcal{J}_{m, n}(a, \lambda)=\sum_{\mu=0}^{m-n} \frac{\log _{2} e}{(m-n-\mu) !}\left[\frac{(-1)^{m-n-\mu-1}}{(a \lambda)^{m-n-\mu}} e^{\frac{1}{a \lambda}} \operatorname{Ei}\left(\frac{-1}{a \lambda}\right)+\sum_{l=1}^{m-n-\mu}(l-1) !\left(\frac{-1}{a \lambda}\right)^{m-n-\mu-l}\right]
$$

Gaussian distributed. ${ }^{2}$ Then, we obtain a lower bound on the achievable rate of the uplink transmission from the $k$ th user as

$$
R_{\mathrm{P}, k}^{\mathrm{MRC}}=\mathbb{E}\left\{\log _{2}\left(1+\frac{\gamma_{\mathrm{u}}\left\|\boldsymbol{g}_{k}\right\|^{2}}{\gamma_{\mathrm{u}} \sum_{i \neq k}^{K} \beta_{i}+1}\right)\right\}
$$

where $\gamma_{\mathrm{u}} \triangleq p_{\mathrm{u}} / \sigma^{2}$.

Since $\boldsymbol{g}_{k}$ is an $M \times 1$ Gaussian vector whose elements are independent with zero mean and variance $\beta_{k}$, we can use Lemma 1 in the appendix to obtain

$$
R_{\mathrm{P}, k}^{\mathrm{MRC}}=\mathcal{J}_{M, 1}\left(\frac{\gamma_{\mathrm{u}}}{\gamma_{\mathrm{u}} \sum_{i \neq k}^{K} \beta_{i}+1}, \beta_{k}\right)
$$

where $\mathcal{J}_{m, n}(a, \lambda)$ given by (28) shown at the top of the page, where $\operatorname{Ei}(\cdot)$ is the exponential integral function $[14$, eq. (8.211.1)].

If $p_{\mathrm{u}}=E_{\mathrm{u}} / M$, and $M$ grows without bound, then from (26), we have

$$
\begin{aligned}
R_{\mathrm{P}, k}^{\mathrm{MRC}} & =\mathbb{E}\left\{\log _{2}\left(1+\frac{E_{\mathrm{u}}\left\|\boldsymbol{g}_{k}\right\|^{2} / M}{E_{\mathrm{u}}\left(\sum_{i \neq k}^{K} \beta_{i}\right) / M+\sigma^{2}}\right)\right\} \\
& \rightarrow \log _{2}\left(1+\frac{\beta_{k} E_{\mathrm{u}}}{\sigma^{2}}\right), M \rightarrow \infty
\end{aligned}
$$

which equals the rate value obtained from Proposition 1. This is due to the fact that when $M \rightarrow \infty$, the interefence part is canceled and hence, the bound converges to the exact value.

2) Zero-Forcing Receiver: From (9), with perfect CSI, the received signal after applying the $\mathrm{ZF}$ scheme becomes

$$
r_{\mathrm{ZF}}=\sqrt{p_{\mathrm{u}}} \boldsymbol{x}+\boldsymbol{G}^{\dagger} \boldsymbol{n}
$$

Therefore, the uplink achievable rate from the $k$ th user is given by

$$
R_{\mathrm{P}, k}^{\mathrm{ZF}}=\mathbb{E}\left\{\log _{2}\left(1+\frac{\gamma_{\mathrm{u}}}{\left[\left(\boldsymbol{G}^{H} \boldsymbol{G}\right)^{-1}\right]_{k k}}\right)\right\} .
$$

From Lemma 2 in the appendix, we obtain

$$
R_{\mathrm{P}, k}^{\mathrm{ZF}}=\mathcal{J}_{M, K}\left(\gamma_{\mathrm{u}}, \beta_{k}\right) .
$$

\footnotetext{
${ }^{2}$ From (8) and (25), the assumption that the interference plus noise is Gaussian distributed is true when $K$ is large.
}

If $p_{\mathrm{u}}=E_{\mathrm{u}} / M,(31)$ can be rewritten as

$$
\begin{aligned}
R_{\mathrm{P}, k}^{\mathrm{ZF}} & =\mathbb{E}\left\{\log _{2}\left(1+\frac{E_{\mathrm{u}} / M}{\frac{\sigma^{2}}{M \beta_{k}}\left[\left(\frac{\boldsymbol{H}^{H} \boldsymbol{H}}{M}\right)^{-1}\right]_{k k}}\right)\right\} \\
& \rightarrow \log _{2}\left(1+\frac{\beta_{k} E_{\mathrm{u}}}{\sigma^{2}}\right), M \rightarrow \infty .
\end{aligned}
$$

The results in (29) and (33) show again that when $M \rightarrow \infty$, the performance of ZF approaches that of MRC.

\section{B. Imperfect Channel State Information}

1) Maximum-Ratio Combining: Denote by $\mathcal{E} \triangleq \hat{\boldsymbol{G}}-\boldsymbol{G}$. Then, from (16), the elements of the $i$ th column of $\mathcal{E}$ are Gaussian RVs with zero means and variances $\frac{\beta_{i} \sigma^{2}}{p_{\mathrm{p}} \beta_{i}+\sigma^{2}}$. Furthermore, from the properties of MMSE estimation, $\mathcal{E}$ is independent of $\hat{G}$. The received vector at the base station can be rewritten as

$$
\hat{\boldsymbol{r}}_{\mathrm{MRC}}=\hat{\boldsymbol{G}}^{H}\left(\sqrt{p_{\mathrm{u}}} \hat{\boldsymbol{G}} \boldsymbol{x}-\sqrt{p_{\mathrm{u}}} \mathcal{E} \boldsymbol{x}+\boldsymbol{n}\right) .
$$

Therefore, after using MRC, the received signal transmitted from the $k$ th user is

$$
\begin{aligned}
\hat{r}_{\mathrm{MRC}, k}=\sqrt{p_{\mathrm{u}}}\left\|\hat{\boldsymbol{g}}_{k}\right\|^{2} x_{k} & +\sqrt{p_{\mathrm{u}}} \sum_{i \neq k}^{K} \hat{\boldsymbol{g}}_{k}^{H} \hat{\boldsymbol{g}}_{i} x_{i} \\
& -\sqrt{p_{\mathrm{u}}} \sum_{i=1}^{K} \hat{\boldsymbol{g}}_{k}^{H} \boldsymbol{\varepsilon}_{i} x_{i}+\hat{\boldsymbol{g}}_{k}^{H} \boldsymbol{n}
\end{aligned}
$$

where $\hat{\boldsymbol{g}}_{i}$ and $\varepsilon_{i}$ are the $i$ th columns of $\hat{\boldsymbol{G}}$ and $\mathcal{E}$, respectively. From (16), each element of $\hat{\boldsymbol{g}}_{i}$ is Gaussian distributed with zero mean and variance $\frac{p_{\mathrm{p}} \beta_{i}^{2}}{p_{\mathrm{p}} \beta_{i}+\sigma^{2}}$. Similarly, by dividing the left and right hand sides of (35) by $\left\|\hat{\boldsymbol{g}}_{k}\right\|$, we obtain

$$
\frac{\hat{r}_{\mathrm{MRC}, k}}{\left\|\hat{\boldsymbol{g}}_{k}\right\|}=\sqrt{p_{\mathrm{u}}}\left\|\hat{\boldsymbol{g}}_{k}\right\| x_{k}+\sqrt{p_{\mathrm{u}}} \sum_{i \neq k}^{K} \hat{\tilde{g}}_{i} x_{i}-\sqrt{p_{\mathrm{u}}} \sum_{i=1}^{K} \tilde{\varepsilon}_{i} x_{i}+\hat{\tilde{n}}_{k}
$$

where

$$
\begin{aligned}
& \hat{\tilde{g}}_{i} \triangleq \frac{\hat{\boldsymbol{g}}_{k}^{H} \hat{\boldsymbol{g}}_{i}}{\left\|\hat{\boldsymbol{g}}_{k}\right\|} \sim \mathcal{C N}\left(0, \frac{p_{\mathrm{p}} \beta_{i}^{2}}{p_{\mathrm{p}} \beta_{i}+\sigma^{2}}\right) \\
& \tilde{\varepsilon}_{i} \triangleq \frac{\hat{\boldsymbol{g}}_{k}^{H} \varepsilon_{i}}{\left\|\hat{\boldsymbol{g}}_{k}\right\|} \sim \mathcal{C N}\left(0, \frac{\beta_{i} \sigma^{2}}{p_{\mathrm{p}} \beta_{i}+\sigma^{2}}\right)
\end{aligned}
$$

and $\hat{\tilde{n}}_{k} \triangleq \frac{\hat{\boldsymbol{g}}_{k}^{H} \boldsymbol{n}}{\left\|\hat{\boldsymbol{g}}_{k}\right\|} \sim \mathcal{C N}\left(0, \sigma^{2}\right)$. The base station treats the channel estimate as the true channel, and the part including the last three terms of (36) is considered as noise. Under the assumption that the noise is Gaussian distributed, we obtain a 


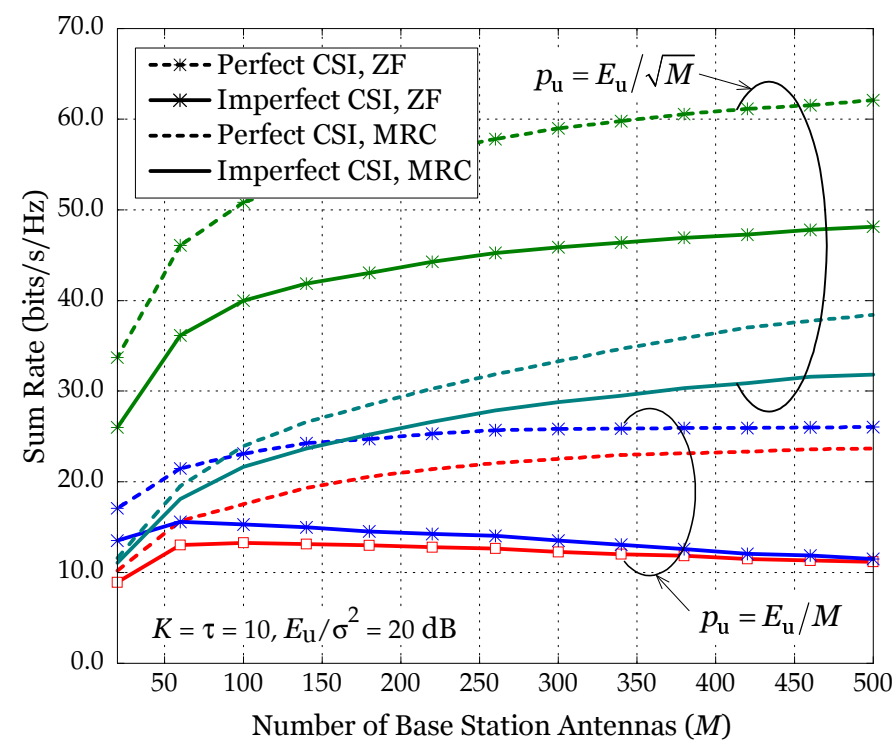

Fig. 1. Lower bound on the uplink sum-rate versus the number of base station antennas. Here $K=10, E_{\mathrm{u}} / \sigma^{2}=20 \mathrm{~dB}$.

lower bound of the uplink achievable rate from the $k$ th user as

$$
R_{\mathrm{IP}, k}^{\mathrm{MRC}}=\mathbb{E}\left\{\log _{2}\left(1+\frac{\gamma_{\mathrm{u}}\left\|\hat{\boldsymbol{g}}_{k}\right\|^{2}}{\gamma_{\mathrm{u}} \sum_{i \neq k}^{K} \beta_{i}+\frac{\gamma_{\mathrm{u}} \beta_{k}}{\tau \gamma_{\mathrm{u}} \beta_{k}+1}+1}\right)\right\} .
$$

Then, again using Lemma 1 in the appendix, we obtain

$$
R_{\mathrm{IP}, k}^{\mathrm{MRC}}=\mathcal{J}_{M, 1}\left(\frac{\gamma_{\mathrm{u}}}{\gamma_{\mathrm{u}} \sum_{i \neq k}^{K} \beta_{i}+\frac{\gamma_{\mathrm{u}} \beta_{k}}{\tau \gamma_{\mathrm{u}} \beta_{k}+1}+1}, \frac{\tau \gamma_{\mathrm{u}} \beta_{k}^{2}}{\tau \gamma_{\mathrm{u}} \beta_{k}+1}\right) .
$$

By choosing $p_{\mathrm{u}}=E_{\mathrm{u}} / \sqrt{M}$ and substituting $\hat{\boldsymbol{g}}_{k}=$ $\frac{p_{\mathrm{p}} \beta_{i}^{2}}{p_{\mathrm{p}} \beta_{i}+\sigma^{2}} \tilde{\boldsymbol{g}}_{k}$, where $\tilde{\boldsymbol{g}}_{k} \sim \mathcal{C N}\left(0, \boldsymbol{I}_{M}\right)$ into (37), and using the law of large numbers, we obtain

$$
R_{\mathrm{IP}, k}^{\mathrm{MRC}} \rightarrow \log _{2}\left(1+\frac{\tau \beta_{k}^{2} E_{\mathrm{u}}^{2}}{\sigma^{4}}\right), M \rightarrow \infty .
$$

Again, we see that when $M \rightarrow \infty$, the asymptotic bound on the rate equals the exact rate value obtained from Proposition 2.

2) Zero-Forcing Receiver: After using the ZF scheme, the received vector at the base station can be rewritten as

$$
\begin{aligned}
\hat{\boldsymbol{r}}_{\mathrm{ZF}} & =\hat{\boldsymbol{G}}^{\dagger}\left(\sqrt{p_{\mathrm{u}}} \hat{\boldsymbol{G}} \boldsymbol{x}-\sqrt{p_{\mathrm{u}}} \mathcal{E} \boldsymbol{x}+\boldsymbol{n}\right) \\
& =\sqrt{p_{\mathrm{u}}} \boldsymbol{x}-\sqrt{p_{\mathrm{u}}} \hat{\boldsymbol{G}}^{\dagger} \mathcal{E} \boldsymbol{x}+\hat{\boldsymbol{G}}^{\dagger} \boldsymbol{n} .
\end{aligned}
$$

Under the assumption that the noise is Gaussian distributed, we obtain a lower bound of the uplink achievable rate from the $k$ th user as

$$
R_{\mathrm{IP}, k}^{\mathrm{ZF}}=\mathbb{E}\left\{\log _{2}\left(1+\frac{\gamma_{\mathrm{u}}}{\left(\sum_{i=1}^{K} \frac{\gamma_{\mathrm{u}} \beta_{i}}{\tau \gamma_{\mathrm{u}} \beta_{i}+1}+1\right)\left[\left(\hat{\boldsymbol{G}}^{H} \hat{\boldsymbol{G}}\right)^{-1}\right]_{k k}}\right)\right\}
$$

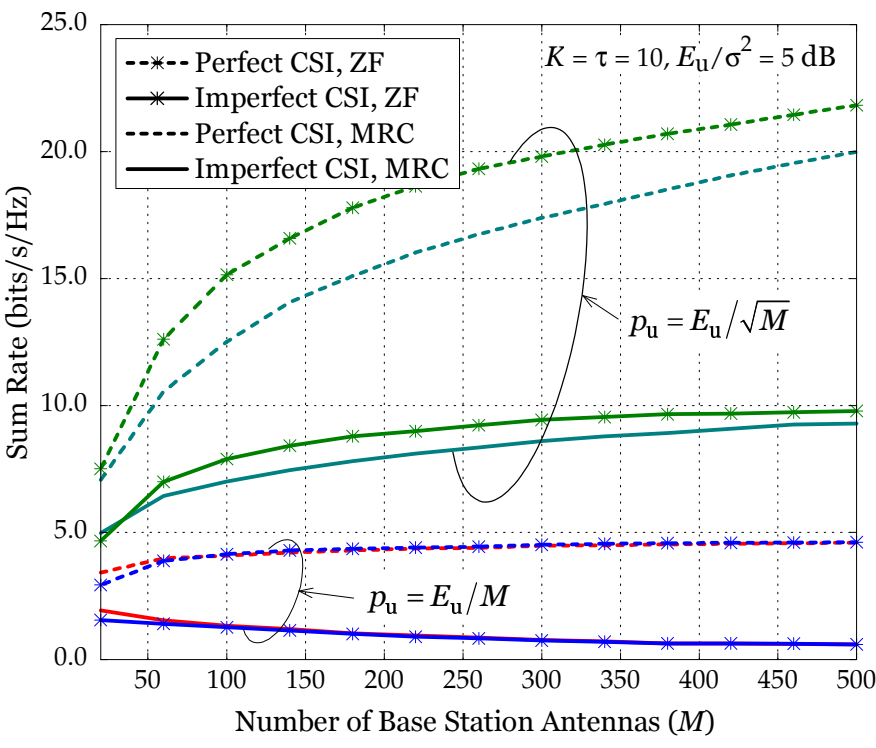

Fig. 2. Lower bound on the uplink sum-rate versus the number of base station antennas. Here $K=10, E_{\mathrm{u}} / \sigma^{2}=5 \mathrm{~dB}$.

From Lemma 1 in the appendix, we obtain

$$
R_{\mathrm{IP}, k}^{\mathrm{ZF}}=\mathcal{J}_{M, K}\left(\frac{\gamma_{\mathrm{u}}}{\gamma_{\mathrm{u}} \sum_{i=1}^{K} \frac{\beta_{i}}{\tau \gamma_{\mathrm{u}} \beta_{i}+1}+1}, \frac{\tau \gamma_{\mathrm{u}} \beta_{k}^{2}}{\tau \gamma_{\mathrm{u}} \beta_{k}+1}\right) .
$$

Similarly, with $p_{\mathrm{u}}=E_{\mathrm{u}} / \sqrt{M}$, when $M \rightarrow \infty$, the bound on the uplink achievable rate for ZF tends to the one for MRC (see (39)), i.e.,

$$
R_{\mathrm{IP}, k}^{\mathrm{ZF}} \rightarrow \log _{2}\left(1+\frac{\tau \beta_{k}^{2} E_{\mathrm{u}}^{2}}{\sigma^{4}}\right), M \rightarrow \infty
$$

which equals the rate value obtained from Proposition 2.

\section{Numerical Results}

We consider a hexagonal cellular network with a radius (from center to vertex) of 1000 meters. The number of users is $K=10$. The users are located uniform randomly in the cell and we assume that no user is closer to the base station than $r_{\mathrm{h}}=100$ meters. The large-scale fading $\beta_{k}$ is modelled via $z_{k} /\left(r_{k} / r_{\mathrm{h}}\right)^{\nu}$, where $z_{k}$ is a log-normal random variable with standard deviation $\sigma_{\text {shadow }}, r_{k}$ is the distance between the $k$ th user and the base station, and $\nu$ is the path loss exponent. For all examples, we choose $\sigma_{\text {shadow }}=8 \mathrm{~dB}$, and $\nu=2.2$. For CSI estimated from uplink pilots, we choose pilot sequences with length $\tau=K$. (This is the smallest amount of training that can be used.)

Fig. 1 shows the lower bounds (27), (32), (38), and (42) on the uplink sum-rates versus the number of base station antennas for $p_{\mathrm{u}}=E_{\mathrm{u}} / M$ and $p_{\mathrm{u}}=E_{\mathrm{u}} / \sqrt{M}$ with perfect and imperfect receiver CSI, and with $\mathrm{MRC}$ and $\mathrm{ZF}$, respectively. Here, we choose $E_{\mathrm{u}} / \sigma^{2}=20 \mathrm{~dB}$. At this SNR, the sumrate is in the order of $10-60 \mathrm{bits} / \mathrm{s} / \mathrm{Hz}$, corresponding to a spectral efficiency per user of $1-6 \mathrm{bits} / \mathrm{s} / \mathrm{Hz}$. While the SNR operating point here was chosen somewhat arbitrarily, the operating points are reasonable from a practical point of view. 
For example, 64-QAM with a rate-3/4 channel code would correspond to $4.5 \mathrm{bits} / \mathrm{s} / \mathrm{Hz}$. (Figure 2, see below, shows results at lower SNR.)

As expected, with $p_{\mathrm{u}}=E_{\mathrm{u}} / M$, when $M$ increases, the sum-rate converges to a constant value for the case of perfect CSI, while the sum-rate decreases to 0 for the case of imperfect CSI. However, with $p_{\mathrm{u}}=E_{\mathrm{u}} / \sqrt{M}$, for the imperfect CSI case, the sum-rate converges to a constant value instead of zero as $M$ increases, and for the perfect CSI case, the sumrate grows without bound as $M \rightarrow \infty$. The growth rate is logarithmic in the received power and therefore logarithmic in the number of antennas.

These numerical results show that we can reduce the transmitted power of each user to $E_{\mathrm{u}} / M$ for the perfect CSI case, and to $E_{\mathrm{u}} / \sqrt{M}$ for the imperfect CSI case when $M$ is large. Typically ZF is better than MRC at high SNR, and vice versa at low SNR [11]. When comparing MRC and ZF in Figure 1, we see that here, when the transmitted power is inversely proportional to $\sqrt{M}$, the power is not low enough to make MRC perform as well as ZF. But when the transmitted power is inversely proportional to $M$, we see that MRC performs almost as well as ZF for large $M$.

In Fig. 2, we consider the same setting as Fig. 1, but we choose $E_{\mathrm{u}} / \sigma^{2}=5 \mathrm{~dB}$. This figure provides the same insights as Fig. 2. We can see that, the gap between the performance of MRC and the performance of ZF is reduced compared with Fig. 1. This is due to the fact that, ZF works well at high power, while MRC is better at low power.

We next consider the required transmit power of each user that is needed to reach a fixed spectral efficiency. Fig. 3 shows the normalized power $\left(p_{\mathrm{u}} / \sigma^{2}\right)$ required to achieve 1 $\mathrm{bit} / \mathrm{s} / \mathrm{Hz}$ per user for different $M$. The asymptotic curves are determined from Equations (29) for the case of perfect CSI, and (39) for the case of imperfect CSI. As expected, for large number of base station antennas, by doubling $M$, we can gain approximately $3 \mathrm{~dB}$ and $1.5 \mathrm{~dB}$ in power with perfect CSI and imperfect CSI, respectively. Furthermore, we can see that when $M$ is large, i.e., $M / K>6$, the difference in performance between MRC and ZF is less than $1 \mathrm{~dB}$.

\section{CONCLUSION}

We studied the uplink transmission of data from $K$ autonomous terminals to a compact array of $M$ antennas with respect to power-efficiency and throughput. We found that power-efficiency is qualitatively different depending on whether the receiver has perfect channel-state information (CSI) or whether it has only an imperfect channel estimate that is derived from uplink pilots. With perfect CSI the radiated power of the terminals can be made inversely proportional to $M$ while maintaining spatial multiplexing gains, while for imperfect CSI the power can only be made inversely proportional to the square-root of $M$. We also compared two receiver structures: zero-forcing (ZF) and maximum-ratio combining (MRC). Except for the case of imperfect channel knowledge and a small number of antennas, ZF outperforms MRC. However the "green" activity of reducing radiated

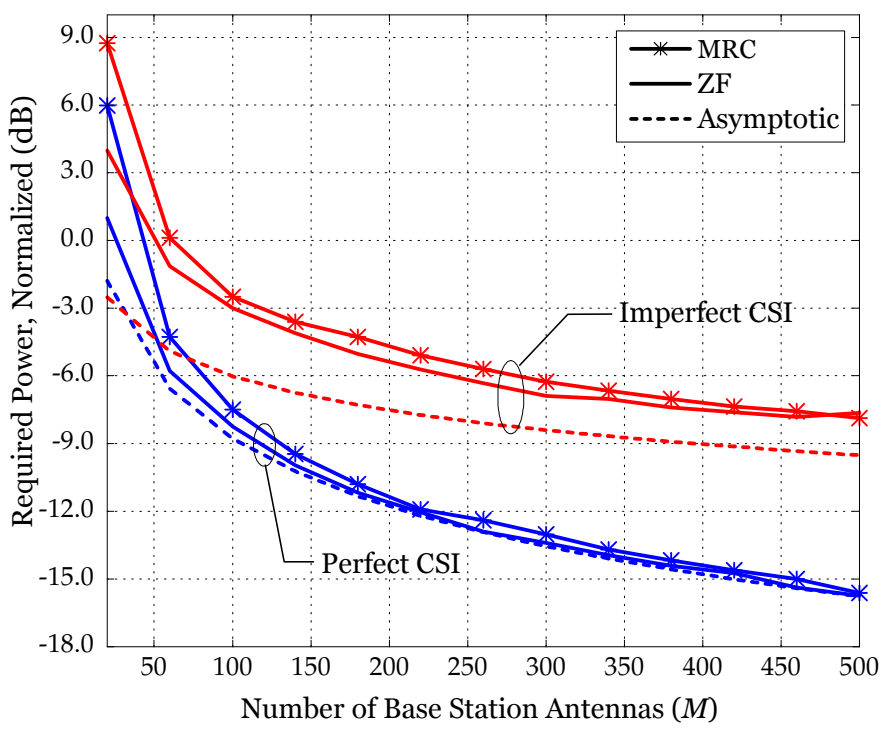

Fig. 3. Required power versus the number of base station antennas for 1 bit/s/Hz per user. Here $K=10$.

power as the number of service antennas increases narrows the performance gap between MRC and ZF. In short, a large excess of antennas yields high spectral efficiency, high powerefficiency, with low-complexity decentralized processing.

\section{APPENDIX}

Here we state and prove two lemmas which are used to derive the closed-form expressions of the achievable rates in Section IV.

Lemma 1: Let $z$ be an $m \times 1$ Gaussian vector whose elements are independent with zero mean and variance $\sigma_{z}^{2}$. Then

$$
\mathbb{E}\left\{\log _{2}\left(1+a\|z\|^{2}\right)\right\}=\mathcal{J}_{m, 1}\left(a, \sigma_{z}^{2}\right)
$$

where $\mathcal{J}_{m, n}(a, \lambda)$ is given by (28).

Proof: Define $Z \triangleq\|\boldsymbol{z}\|^{2}$. Then $\frac{2}{\sigma^{2}} Z$ has a Chi-square distribution with $2 m$ degrees of freedom. Therefore, the probability density function (PDF) of $Z$ is given by

$$
p_{Z}(z)=\frac{1}{(m-1) ! \sigma_{z}^{2 m}} z^{m-1} e^{-z / \sigma_{z}^{2}}, z \geq 0 .
$$

Using (45), (44) can be rewritten as

$$
\begin{aligned}
\mathbb{E}\left\{\log _{2}\left(1+a\|\boldsymbol{z}\|^{2}\right)\right\} & =\frac{1}{(m-1) ! \sigma_{z}^{2 m}} \\
& \times \int_{0}^{\infty} \log _{2}(1+a z) z^{m-1} e^{-z / \sigma_{z}^{2}} d z .
\end{aligned}
$$

Using [14, eq. (4.337.5)], we obtain (44).

Lemma 2: Let $X$ be an $m \times n$ random matrix whose rows are zero-mean independent Gaussian vectors with covariance matrix $\Sigma(m \geq n)$. Then

$$
\mathbb{E}\left\{\log _{2}\left(1+\frac{a}{\left[\left(\boldsymbol{X}^{H} \boldsymbol{X}\right)^{-1}\right]_{k k}}\right)\right\}=\mathcal{J}_{m, n}\left(a, \boldsymbol{\Sigma}_{k k}\right)
$$


Proof: Let

$$
\gamma_{k} \triangleq \frac{a}{\left[\left(\boldsymbol{X}^{H} \boldsymbol{X}\right)^{-1}\right]_{k k}}
$$

Then the PDF of $\gamma_{k}$ is given by [15]

$$
p_{\gamma_{k}}(\gamma)=\frac{e^{-\boldsymbol{\Sigma}_{k k}^{-1} \gamma / a}}{(m-n) ! a \boldsymbol{\Sigma}_{k k}}\left(\frac{\gamma}{a \boldsymbol{\Sigma}_{k k}}\right)^{m-n} .
$$

Applying (49) into (47), we obtain

$$
\begin{aligned}
& \mathbb{E}\left\{\log _{2}\left(1+\frac{a}{\left[\left(\boldsymbol{X}^{H} \boldsymbol{X}\right)^{-1}\right]_{k k}}\right)\right\} \\
& =\int_{0}^{\infty} \log _{2}(1+\gamma) \frac{e^{-\boldsymbol{\Sigma}_{k k}^{-1} \gamma / a}}{(m-n) ! a \boldsymbol{\Sigma}_{k k}}\left(\frac{\gamma}{a \boldsymbol{\Sigma}_{k k}}\right)^{m-n} d \gamma .
\end{aligned}
$$

Using [14, eq. (4.337.5)], we obtain (47).

\section{ACKNOWLEDGMENT}

This work was supported in part by the Swedish Research Council (VR), the Swedish Foundation for Strategic Research (SSF), and ELLIIT. E. Larsson is a Royal Swedish Academy of Sciences (KVA) Research Fellow supported by a grant from the Knut and Alice Wallenberg Foundation.

\section{REFERENCES}

[1] D. Gesbert, M. Kountouris, R. W. Heath Jr., C.-B. Chae, and T. Sälzer, "Shifting the MIMO paradigm," IEEE Sig. Proc. Mag., vol. 24, no. 5, pp. 36-46, 2007.

[2] G. Caire, N. Jindal, M. Kobayashi, and N. Ravindran, "Multiuser MIMO achievable rates with downlink training and channel state feedback," IEEE Trans. Inf. Theory, vol. 56, no. 6, pp. 2845-2866, 2010.

[3] J. Jose, A. Ashikhmin, T. L. Marzetta, and S. Vishwanath, "Pilot contamination problem in multi-cell TDD systems," in Proc. IEEE International Symposium on Information Theory (ISIT'09), Seoul, Korea, Jun. 2009, pp. 2184-2188.

[4] S. Verdú, "Computational complexity of multiuser detection," Algorithmica., vol. 4, pp. 303-312, 1989.

[5] P. Viswanath and D. N. C. Tse, "Sum capacity of the vector Gaussian broadcast channel and uplink-downlink duality" IEEE Trans. Inf. Theory, vol. 49, no. 8, pp. 1912-1921, Aug. 2003.

[6] H. Weingarten, Y. Steinberg, and S. Shamai, "The capacity region of the Gaussian multiple-input multiple-output broadcast channel," IEEE Trans. Inf. Theory, vol. 52, no. 9, pp. 3936-3964, Sep. 2006.

[7] T. L. Marzetta, "Noncooperative cellular wireless with unlimited numbers of base station antennas," IEEE Trans. Wireless Commun., vol. 9, no. 11, pp. 3590-3600, Nov. 2010.

[8] H. Q. Ngo, T. L. Marzetta, and E. G. Larsson, "Analysis of the pilot contamination effect in very large multicell multiuser MIMO systems for physical channel models," in Proc. IEEE International Conference on Acoustics, Speech and Signal Processing (ICASSP'11), Prague, Czech Republic, May 2011, pp. 3464-3467.

[9] T. L. Marzetta, "How much training is required for multiuser MIMO," in Fortieth Asilomar Conference on Signals, Systems and Computers (ACSSC '06), Pacific Grove, CA, USA, Oct. 2006, pp. 359-363.

[10] E. G. Larsson and P. Stoica, Space-Time Block Coding for Wireless Communication. Cambridge, UK: Cambridge University Press, 2003.

[11] D. N. C Tse and P. Viswanath, Fundamentals of Wirless Communications. Cambridge, UK: Cambridge University Press, 2005.

[12] H. Cramér, Random Variables and Probability Distributions. Cambridge, UK: Cambridge University Press, 1970.

[13] B. Hassibi and B. M. Hochwald, "How much training is needed in multiple-antenna wireless links?" IEEE Trans. Inf. Theory, vol. 49, no. 4, pp. 951-963, Apr. 2003.
[14] I. S. Gradshteyn and I. M. Ryzhik, Table of Integrals, Series, and Products, 7th ed. San Diego, CA: Academic, 2007.

[15] D. A. Gore, R. W. Heath Jr., and A. J. Paulraj, "Transmit selection in spatial multiplexing systems," IEEE Commun. Lett., vol. 6, no. 11, pp. 491-493, Nov. 2002. 